\title{
Complex spatial organization in a simple model of resource allocation
}

\author{
Damián H. Zanette \\ Consejo Nacional de Investigaciones Cientificas y Técnicas, \\ Centro Atómico Bariloche and Insituto Balseiro, 8400 Bariloche, Río Negro, Argentina
}

(October 30, 2018)

\begin{abstract}
A dynamical model for the distribution of resources between competing agents is studied. While global competition leads to the accumulation of all the resources by a single agent, local competition allows for a wider resource distribution. Multiplicative processes give rise to almost-ordered spatial structures, thourgh the enhancement of random fluctuations
\end{abstract}

PACS numbers: 87.23.Ge, 87.23.Cc, 05.65.+b

Though many real systems in the scopes of biology and the social sciences are well described by agent-based models with pair interactions [1 3], qualitatively similar to the physical description of interacting particles, a large class of biological and social processes are driven by interactions mediated by an external actor, which generally bears no resemblance with the individual agents. This kind of processes - which, in connection with physics, may be assimilated to the evolution of globally coupled dynamical systems 任 - is typically found in systems where agents compete for resources. A particularly relevant instance of this situation is given by competing biological species. In this case, in fact, the interaction is rarely given by struggling events between individuals of different species, but rather by accessing simultaneously to the limited resources provided by the environment. The performance of each agent, i.e. of each species, is here measured by its ability to get resources, which depends both on the capabilities of each individual and on global features such as the total population of the species. The same scenario is found in some economical systems, for instance, in companies competing for financial resources, usually administrated by banks, or even in scientific research projects competing for funds from a government agency. A key ingredient in the dynamics of these systems is that the ability of each agent to get resources at a given time depends, often strongly, on the resources assigned to the agent at previous stages. This can give rise to a multiplicative process that, in the absence of buffer mechanisms, leads to resource accumulation by a single agent. In the following, we consider a simple dynamical model that incorporates these elements and, in particular, study the effects of competition at local level. We find that local competition softens the process of resource accumulation and gives rise, through the enhancement of spatial fluctuations, to nontrivial structures.
Our system consists of an ensemble of $N$ agents, each of them characterized by its productivity $\alpha_{i}$. For future convenience, we consider the generic situation where all productivities are different. At each time step $t$, each agent is assigned an amount of resources $r_{i}(t)$, which is used to produce an amount of products $p_{i}(t)=$ $\alpha_{i} r_{i}(t)$. At the next time step, resources are distributed among agents in amounts proportional to their production, namely,

$$
r_{i}(t+1)=\frac{p_{i}(t)}{\sum_{j} p_{j}(t)} R(t+1)=\frac{\alpha_{i} r_{i}(t)}{\sum_{j} \alpha_{j} r_{j}(t)} R(t+1),
$$

where $R(t+1)$ are the total resources available at time $t+1$. Model (11) can be fully solved for arbitrary productivities and initial conditions $r_{i}(0)$. In the first place, we note that rescaling $r_{i}(t) / R(t) \rightarrow r_{i}(t)$, Eq. (11) becomes independent of the total resources. We thus fix $R(t)=1$ for all $t$, so that $\sum_{j} r_{j}(t)=1$. In this situation, the solution to Eq. (11) reads

$$
r_{i}(t)=\frac{\alpha_{i}^{t} r_{i}(0)}{\sum_{j} \alpha_{j}^{t} r_{j}(0)}
$$

For asymptotically large times, $r_{i}(t) \rightarrow 0$ for all $i$, except for the agent with the maximal productivity, $\alpha_{\max }=$ $\max _{i}\left\{\alpha_{i}\right\}$, which receives all the available resources. Due to the multiplicative effect of resource allocation according to production, all resources are in the long run accumulated by the agent with the maximal productivity, giving rise to a sort of winner-takes-all state [3]. By analogy with population dynamics we say that the remaining agents become extinct. In fact, in connection with biological populations, this result is a realization of a wellknown principle of ecology, namely, the principle of competitive exclusion [5]1: in a system of biological species competing for the same resources, only one survives and the others undergo extinction. At moderately large $t$ the resources assigned to each agent are well approximated by $r_{i}(t)=\left(\alpha_{i} / \alpha_{\max }\right)^{t} r_{i}(0) / r_{\max }(0)$. Assuming that the productivities $\alpha_{i}$ are drawn at random from a distribution $P_{\alpha}(\alpha)$ and that, for simplicity, resources are evenly assigned at the beginning, $r_{i}(0)=N^{-1}$ for all $i$, the probability distribution for the individual resources at a given time is

$$
P_{r}\left(r_{i}\right)=\frac{\alpha_{\max }}{r_{i} t} r_{i}^{1 / t} P_{\alpha}\left(\alpha_{\max } r_{i}^{1 / t}\right)
$$


The dependence of this function on $r_{i}$ through the power $r_{i}^{1 / t}$ is very weak for large $t$, so that on a wide interval of the variable we find $P_{r}\left(r_{i}\right) \propto r_{i}^{-1}$. For long times, thus, resources are distributed among agents following a power law with exponent -1 . Compare this result with Pareto's law of wealth distribution [6, 3].

Model (1) admits several variations, that may be of interest in connection with the description of some real systems. For instance, the extinction of all but one agent can be avoided by modifying slightly the allocation method. If, at each time step, a small fraction $\rho$ of the total resources is evenly distributed among the agents whereas the remaining is assigned according to production as above, the individual resources are always larger than $\rho / N$. In this situation, $P_{r}\left(r_{i}\right)$ becomes stationary for long times and, if $\rho$ is small enough, the power-law dependence quoted above is found again for $r_{i}>\rho / N$.

A second variation consists in assuming that the productivity can in turn depend on the individual resources, for instance, as $\alpha_{i}\left(r_{i}\right)=\alpha_{i}^{0} A\left(r_{i}\right)$. If $A(r)$ increases with $r$, the winner-takes-all effect is enhanced. The opposite case, where $A(r)$ is a decreasing function, is more interesting. Lower productivities for higher resources may be the consequence of size effects, crowding, or loss of efficiency due to lower competition. In this case, the system evolves to a stationary situation where the agents whose productivity weights $\alpha_{i}^{0}$ are above a certain threshold $\alpha_{\min }^{0}$ receive nonzero resources, whereas the agents with $\alpha_{i}^{0}<\alpha_{\min }^{0}$ become extinct. In the stationary situation, all the surviving agents have the same productivity $\alpha$. The third instance, where $A(r)$ is a nonmonotonous function, can give origin to multiple stationary nontrivial solutions.

In the following we focus the attention on a variation of model (1) that introduces a spatial distribution of agents. Though the total resources are still allocated among all the ensemble, the agents compete at a local level only. The set of agents that compete with a given agent $i$ defines its neighborhood $\mathcal{N}_{i}$. Resources are assigned according to

$$
r_{i}(t+1)=\frac{1}{Z(t)} \frac{\alpha_{i} r_{i}(t)}{\sum_{j \in \mathcal{N}_{i}} \alpha_{j} r_{j}(t)},
$$

where

$$
Z(t)=\sum_{i} \frac{\alpha_{i} r_{i}(t)}{\sum_{j \in \mathcal{N}_{i}} \alpha_{j} r_{j}(t)}
$$

is a normalization factor that insures that $\sum_{j} r_{j}(t)=1$ for all $t$. We have performed numerical simulations of this system in ensembles of $N=10^{3}$ to $10^{4}$ agents distributed on various geometries. The productivities $\alpha_{i}$ were drawn at random from a uniform distribution in $(0,1)$. Note that, in Eqs. (11) and (4), a homogeneous rescaling of all productivities leaves the models invariant. Several distributions of initial conditions were tested, but there are no essential differences with the case where, at the first step, resources are evenly allocated, $r_{i}(0)=N^{-1}$. Therefore, we concentrate our simulations on this simple case.

In the first place, we consider a one-dimensional array of agents with periodic boundary conditions. The neighborhood of the $i$ th agent consists of its two nearest neighbors. We find that, for long times, approximately half of the agents become extinct and resources are evenly allocated among the surviving agents. Practically everywhere along the array agents are ordered in an alternating sequence of extinct agents and survivors. Occasionally, one finds two neighbor sites where both agents are extinct, but two survivors are never contiguous. In other words, in the neighborhood of a surviving agent no other survivor can be found [cf. model (1)]. The surprising feature of this asymptotic distribution is that the strong correlation between survival and productivity found for Eq. (1) is apparently lost in Eq. (四). In fact, productivities are distributed completely at random along the array, whereas the resulting distribution of survivors is highly ordered. At first glance, no connection can be established, for instance, between survivor sites and local maxima of the productivity or any spatial pattern caused by fluctuations in the distribution of $\alpha_{i}$.

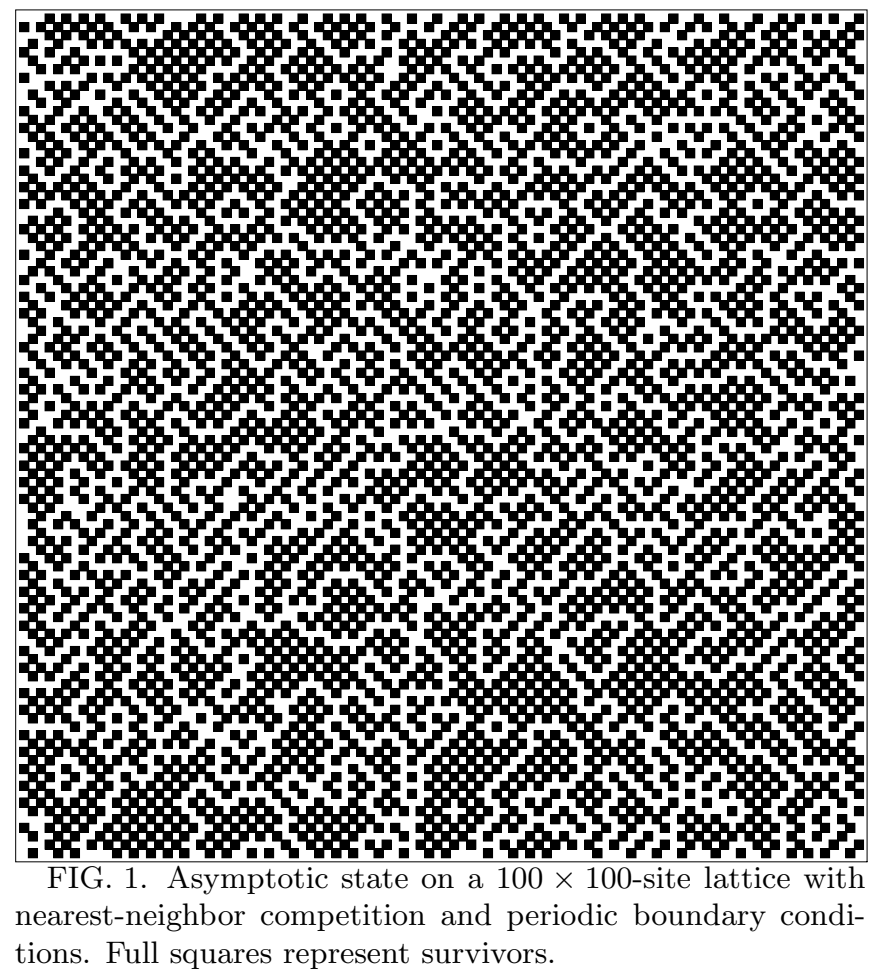

The same feature is found in a two-dimensional array with periodic boundary conditions, where the neighborhood of each site consists of the four nearest neighbors. Figure 11 displays the asymptotic state on a $100 \times 100$ site lattice $\left(N=10^{4}\right)$, where the full squares represent 
survivor sites. Regular domains where survivors and extinct agents alternate in both dimensions, separated by worm-like boundaries formed by extinct agents, are apparent. We have also verified that different definitions of the neighborhood of a site, both in one and in two-dimensional lattices, produce similar almost-periodic asymptotic structures. Again, resources are evenly distributed between the surviving agents and, in neighborhood of a survivor, no other survivor can be found. For instance, in a one-dimensional array where the neighborhood consists of four sites (nearest and next-to-nearest neighbors), the resulting structure is a periodic sequence of two extinct agents and one survivor. Occasional defects, with larger zones of extinction, are also found.

In order to trace the origin of the almost-periodic structures emerging at asymptotically long times we have inspected the successive states of the system from the earliest evolution stages, for the specific case of a onedimensional array of agents with two neighbors per site and periodic boundary conditions. As above, productivities are drawn at random from a uniform distribution and $r_{i}(0)=N^{-1}$ for all $i$. We find that even at the first step of the evolution the distribution of resources already exhibits an almost-periodic sequence of relatively high and low values, in spite of the fact that productivities are spatially uncorrelated. Namely, for a substantial fraction of agents, we have $\left[r_{i+1}(1)-r_{i}(1)\right]\left[r_{i}(1)-r_{i-1}(1)\right]<0$. This observation reveals that the simple dynamics of model (1) is able to introduce strong spatial correlations to an initially uncorrelated state, even at the level of a single evolution step.

To quantify this effect, we take a set of five uncorrelated random numbers $\alpha_{1}, \ldots, \alpha_{5}$ drawn from the productivity distribution, and consider the combinations

$$
\sigma_{i}=\frac{\alpha_{i}}{\alpha_{i-1}+\alpha_{i}+\alpha_{i+1}}
$$

for $i=2,3,4$. The quantities $\sigma_{i}$ are then proportional to the resources received at $t=1$ by three consecutive agents in the array [cf. Eq. (퍼)]. Numerical realizations of these quantities, over $10^{6}$ independent choices of $\alpha_{1}, \ldots, \alpha_{5}$, show that $\left(\sigma_{4}-\sigma_{3}\right)\left(\sigma_{3}-\sigma_{2}\right)<0$ with probability $p_{1}=0.762$. Consequently, slightly more than 76 $\%$ of the agents are expected to belong to a periodic sequence of alternating high and low resources at the first time step. The remaining $24 \%$ stands for defects in the periodic structure. The same kind of analysis can be performed for successive steps in the evolution. It is found that correlations are further enhanced by the dynamics. At the second evolution step, for instance, the probability for an agent to belong to the periodic structure grows to $p_{2}=0.782$. For later stages, we find $p_{5}=0.816$, $p_{10}=0.839, p_{20}=0.854$, and $p_{50}=0.864$. These probabilities saturate at $p_{\infty} \approx 0.865$.

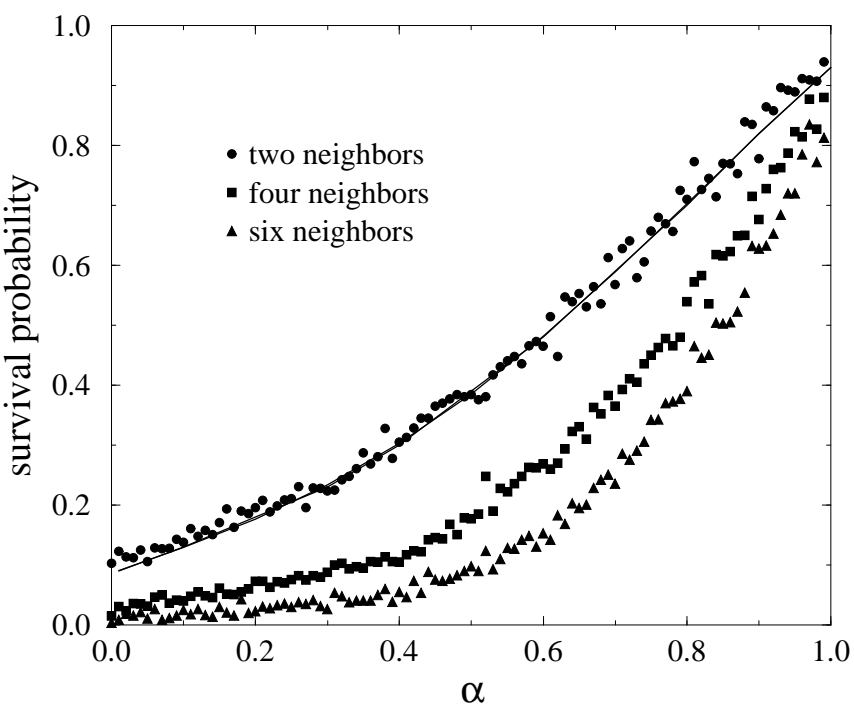

FIG. 2. Survival probability as a function of the productivity $\alpha$ for one dimensional arrays of 1000 agents and different neighborhoods. Dots correspond to average measurements over 100 realizations. The line shows the result of an independent calculation method (see text), for the case of two neighbors.

While the emergence of almost-periodic structures seems to indicate that no correlations subsist in model (11) between survival and productivity, the inspection of the evolution equations as well as of Eq. (6) suggests that some remaining correlation should however exist. In fact, low productivities still imply few resources, so that the minima in the periodic structure should preferably coincide with sites with small $\alpha_{i}$. Moreover, defects in the periodic structures-where extinct agents are found in consecutive sites-should also correspond to low-productivity zones. Such remaining correlation can be characterized by the probability of survival as a function of the productivity, $p_{s}(\alpha)$. This probability is defined as the fraction of agents with productivity $\alpha$ which survive at asymptotically long times. We have numerically measured $p_{s}(\alpha)$, in series of 100 independent realizations of the productivity distribution for ensembles with $N=1000$, for various geometries and neighborhoods. Figure 2 shows the survival probability as a function of $\alpha$ for one-dimensional arrays with different numbers of neighbors. Here, the correlation between survival probability and productivity is apparent. Agents with larger productivities are more likely to survive that those with small $\alpha$. Note, however, that for $\alpha=1$ the survival probability is less than unity, so that even with the maximal productivity there are chances of undergoing extinction. Conversely, for $\alpha \rightarrow 0$ (but $\alpha \neq 0$ ), the survival probability is finite. As the number of neighbors grows the probability is more concentrated towards larger productivities, as expected. In the limit where the neighborhood extends to the whole array the original model (11) is recovered, and the survival probability must vanish except 
for $\alpha=1$.

The survival probability can be calculated independently of the numerical realization of the model, using expressions as in Eq. (6). For a fixed value of $\alpha_{3}$ and different random choices of $\alpha_{1}, \alpha_{2}, \alpha_{4}$ and $\alpha_{5}$, an estimate at the first evolution step of the survival probability of the agent with productivity $\alpha_{3}$ is given by the fraction of instances for which $\sigma_{3}>\sigma_{2}, \sigma_{4}$. Generalizing this procedure for successive time steps, the estimate can be improved by considering later stages in the evolution. The line in Fig. 2 corresponds to this estimate at $t=50$. It shows an excellent agreement with the numerical realizations.

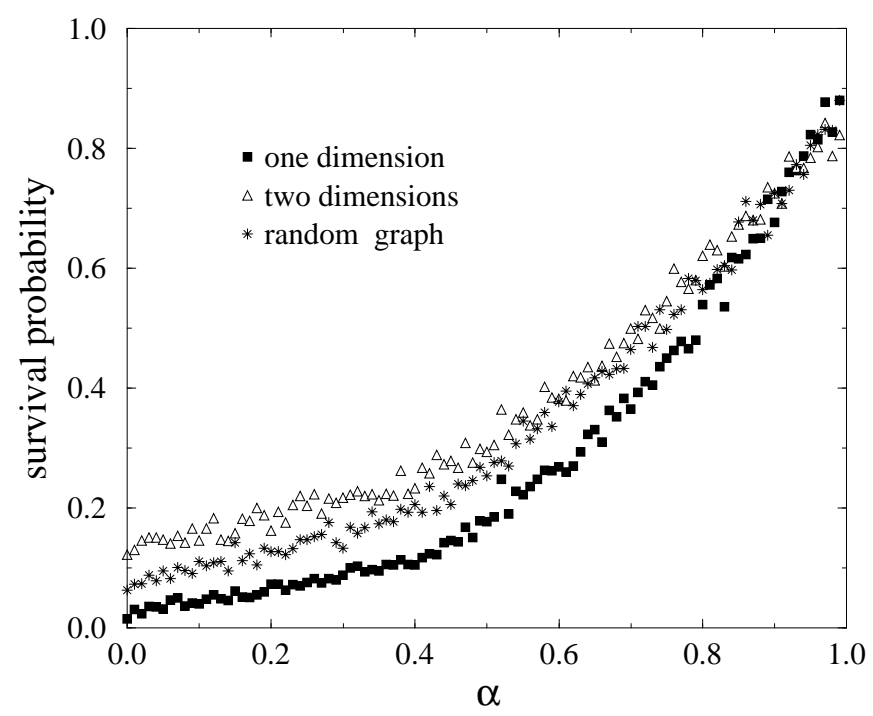

FIG. 3. Survival probability as a function of the productivity $\alpha$ for different geometries with four neighbors per site.

To compare now several geometries, we have measured the survival probability in systems where each agent has the same number of neighbors - specifically, fouron different kinds of arrays, namely, a one-dimensional array (nearest and next-to-nearest neighbors), a twodimensional lattice (nearest neighbors) and a random graph with four connections per site. The results, shown in fig. 3 corresponds to averages over 100 realizations in systems with $N=1000$ for one dimension and random graphs. In this latter case, the graph topology is chosen anew at each realization. For two dimensions, the results correspond to 10 realizations on a $100 \times 100$-array. Though the general trend of $p_{s}(\alpha)$ is qualitatively the same for the three cases, some systematic differences are apparent. For instance, the survival probability in one dimension is appreciably lower than in the other geometries for low productivities. In this range, remarkably, the data for random graphs lies between those for one and two dimensions. For $\alpha>0.8$, instead, the values of $p_{s}(\alpha)$ are hardly distinguishable within our numerical precision.
We may summarize our main results as follows. First, the strong, deterministic correlation between survival and maximal productivity that characterizes the model with global competition (11) is replaced, in the model with local competition (4), by a much weaker, probabilisticlike correlation. Agents with high productivity are more likely to survive but, even with the maximal productivity, extinction cannot be completely discarded. On the other hand, agents with very low productivity have a chance of survival. This conclusion should be relevant to the possible applications of the present models, both to economy and to biology. Second, the loss of the above mentioned deterministic correlation is accompanied by the formation of an almost-regular spatial structure, with a periodic alternation of survivors and extinct agents. This structure is explained by the emergence of strong spatial correlations out of the fully uncorrelated distribution of productivities, due to the very action of the evolution rules. Nontrivial correlations in the conditional probabilities for certain combinations of uncorrelated variablesa rather simple but counterintuitive phenomenon-have recently been pointed out for series of random numbers [7]. The present model illustrates the occurrence of the same kind of phenomenon in a spatially extended dynamical system.

This work has been partially carried out at the Abdus Salam International Centre for Theoretical Physics (Trieste, Italy). The author thanks the Centre for hospitality, and G. Abramson for useful comments.

[1] J. D. Murray, Mathematical Biology (Springer, Berlin, 1989).

[2] P. Bak, S. F. Norrellykke, and M. Shubik, Phys. Rev. E 60, 2528 (1999).

[3] J.-P. Bouchaud and M. Mézard, Physica A 282, 536 (2000).

[4] See, for instance, K. Kaneko, Physica D 41, 137 (1990); N. Nakagawa and Y. Kuramoto, Physica D 75, 74 (1994).

[5] G. F. Gause, The Struggle for Existence (Williams \& Wilkins, Baltimore, 1934).

[6] B. B. Mandelbrot, Fractals and Scaling in Finance (Springer, New York, 1997).

[7] D. Sornette and J. V. Andersen, cond-mat/0001324. 